\title{
Subarachnoid Hemorrhage Caused by a Ruptured Anterior Spinal Artery Aneurysm -Case Report-
}

\author{
Jun KARAKAMA, Kazuhiko NAKAGAWA, \\ Taketoshi MAEHARA, and Kikuo OHNO \\ Department of Neurosurgery, Tokyo Medical and Dental University, Tokyo
}

\begin{abstract}
A 51-year-old man presented with an extremely rare case of intracranial subarachnoid hemorrhage caused by rupture of an anterior spinal artery aneurysm manifesting as disturbance of consciousness following sudden onset of neck pain and numbness of the extremities. Cranial computed tomography revealed subarachnoid hemorrhage, mainly in the posterior fossa. Cerebral angiography studies on admission and on the 4th day demonstrated no definite abnormality as a bleeding source. A ventricular catheter was inserted to treat the acute hydrocephalus, and conservative management was continued during the acute period. Third angiography on the 18th day demonstrated an anterior spinal artery aneurysm at the $\mathrm{C} 1$ level which was considered to be the bleeding site. After conservative treatment, the patient was discharged without neurological deficits. Fourth angiography on the 108th day disclosed spontaneous disappearance of the aneurysm, which was confirmed by the fifth angiography on the 269th day. If subarachnoid hemorrhage of unknown etiology is encountered, spinal artery aneurysm should be considered as the bleeding source. Despite the controversy concerning the treatment strategy, ruptured spinal artery aneurysms can be treated conservatively because of the possibility of spontaneous regression. Follow-up angiography is required to evaluate the natural course of the lesion.
\end{abstract}

Key words: aneurysm, anterior spinal artery, subarachnoid hemorrhage

\section{Introduction}

Spinal artery aneurysms are generally associated with arteriovenous malformations (AVMs) or coarctation of the aorta, whereas isolated spinal artery aneurysms are extremely rare. ${ }^{10,15)}$ Spinal artery aneurysms may manifest as compressive lesions or subarachnoid hemorrhage $(\mathrm{SAH}) .{ }^{10)}$ Only 25 cases of ruptured isolated spinal artery aneurysms have been reported. ${ }^{1-3,5-20)}$ We describe a case of an isolated anterior spinal artery aneurysm manifesting as SAH and then eventually disappeared, as confirmed on follow-up angiography.

\section{Case Report}

A 51-year-old man with untreated hypertension developed disturbance of consciousness following sudden onset of neck pain and numbness of the extremities. He soon progressed to deep coma and suffered cardiopulmonary arrest. After cardiopulmonary resuscitation, he was transferred to our institution. On arrival, he showed no reaction to physical stimuli, and his pupils were equally dilated and fixed. Laboratory examination showed no abnormal findings suggestive of inflammation or infection.

Received December 21, 2009; Accepted April 2, 2010
Autoimmune diseases were excluded because he had no relevant history or specific clinical symptoms.

Cranial computed tomography (CT) revealed SAH, mainly in the posterior fossa (Fig. 1). Magnetic resonance (MR) imaging of the cranium and cervical spine showed no significant findings, including aneurysms, AVMs, or other mass lesions. During the 2 hours after onset, his consciousness level gradually improved. Cerebral digital subtraction angiography (DSA) on admission demonstrated no definite abnormality as a bleeding source. However, the right vertebral artery was not opacified distal to the origin of the posterior inferior cerebellar artery (Fig. 2). A ventricular catheter was inserted to treat the acute hydrocephalus, and strict blood pressure management under deep sedation was maintained to prevent re-bleeding. The second DSA was performed on the 4 th day, but the cause of bleeding remained unidentified. However, the blood flow of the right vertebral artery had improved compared to that on the initial DSA (Fig. 3). The clinical course during the acute period was uneventful, and the third DSA on the 18th day demonstrated an anterior spinal artery aneurysm, which measured $2.5 \mathrm{~mm} \times 1.3 \mathrm{~mm}$, via the right vertebral artery, the flow of which had further improved (Fig. 4). The aneurysm was located at the C1 level, and this lesion was considered the bleeding site.

Conservative medical treatment was selected because 


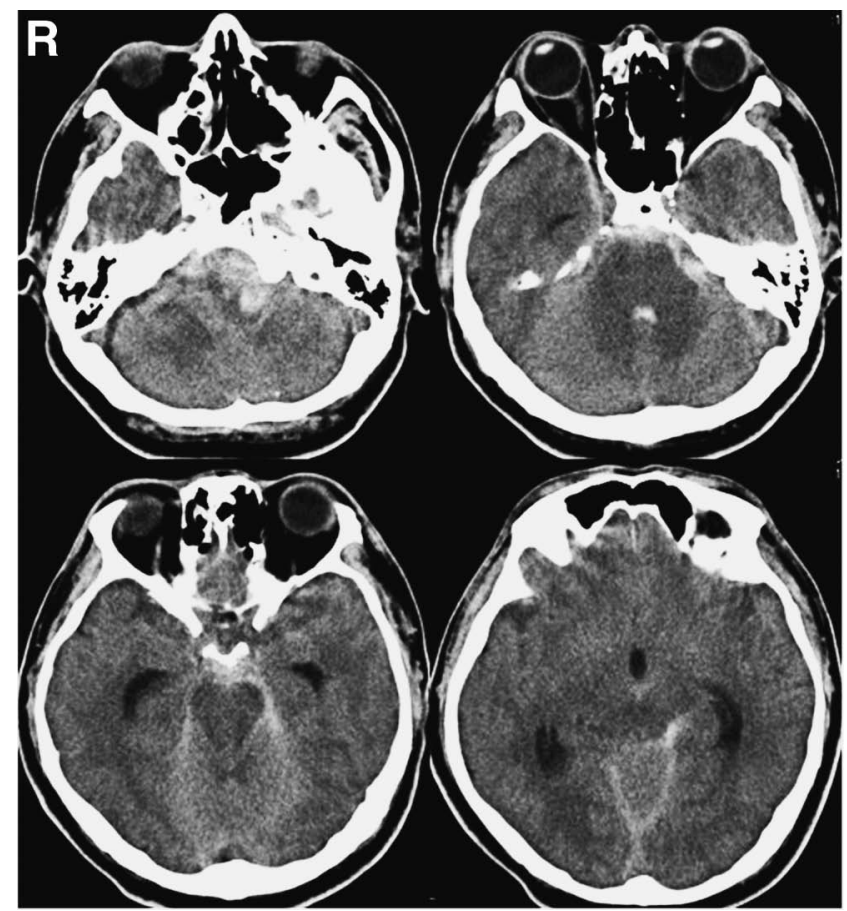

Fig. 1 Cranial computed tomography scans on arrival showing subarachnoid hemorrhage mainly in the posterior fossa.
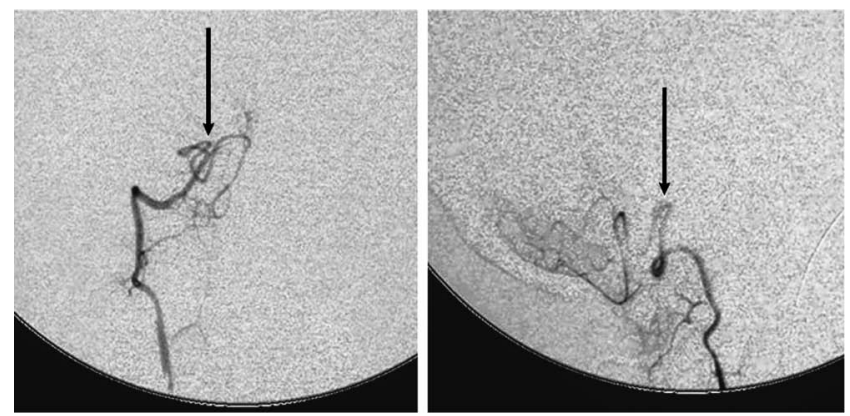

Fig. 2 Right vertebral angiograms on admission, anteroposterior (left) and lateral (right) views, showing insufficient projection of the distal lesion to the origin of the posterior inferior cerebellar artery (arrow).

we thought that the aneurysm would be difficult to obliterate without disturbing the blood flow of the parent artery. The patient eventually became alert and had no neurological deficits. He and his family preferred that he be discharged without the fourth DSA after 62 days of hospitalization. The patient was followed up regularly in the outpatient clinic, and the fourth DSA performed on the 108th day showed no aneurysm (Fig. 5). The fifth DSA on the 269th day confirmed absence of the aneurysm. Therefore, the lesion was considered to have regressed spontaneously.

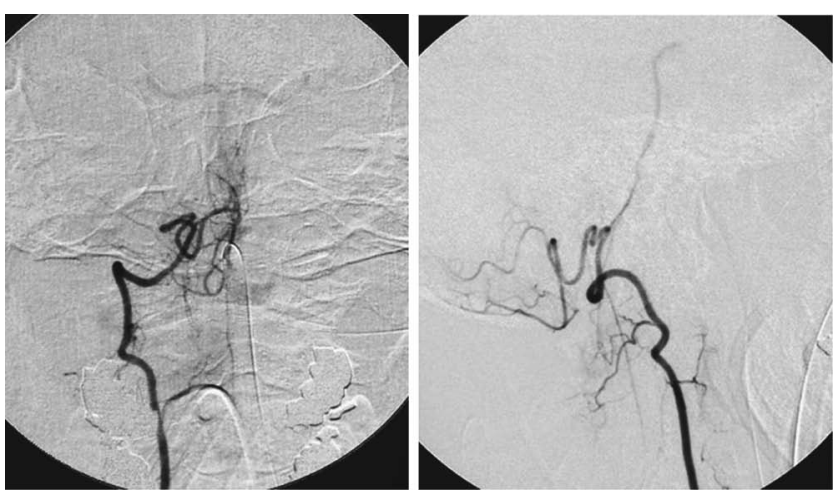

Fig. 3 Right vertebral angiograms on the 4th day, anteroposterior (left) and lateral (right) views, showing improvement of blood flow compared to the initial angiograms.

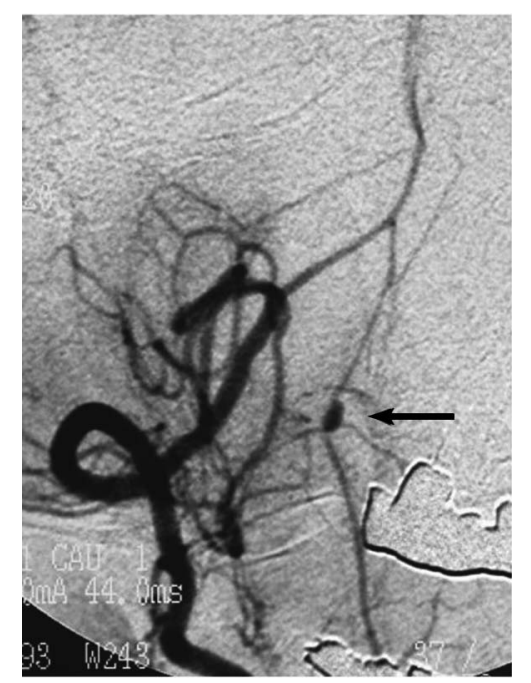

Fig. 4 Right vertebral angiogram on the 18th day revealing a $2.5 \mathrm{~mm} \times 1.3 \mathrm{~mm}$ anterior spinal artery aneurysm (arrow) at the $\mathrm{C} 1$ level.

\section{Discussion}

Only 26 cases of ruptured isolated spinal artery aneurysms, including the present case, have been diagnosed by angiography or at autopsy with clinical information available (Table 1). The mean age of the patients was 51.6 years (range, 29-73 years). There was no sex preponderance, in contrast to the female dominancy found with cerebral artery aneurysms. The aneurysms were mostly located at the upper cervical levels (C1, C2, or C3) and thoracolumbar levels (T11, T12, or L1). Such predilection for specific segmental levels may be because these aneurysms tend to originate from the proximal portion of the anterior spinal artery or the Adamkiewicz artery. Seven of the 26 aneurysms were located on anterior spinal arteries, 5 on Adamkiewicz arteries, and 4 on posterior spinal arteries. If the presence of a spinal artery aneurysm is suspected, concentration on these predominant sites 
may be effective. Additionally, spinal artery aneurysms are less associated with arterial branching sites, unlike cerebral artery aneurysms. ${ }^{1,4,11,15)}$ MR imaging and CT angiography may contribute to the initial diagnosis of spinal artery aneurysms. ${ }^{1,7,11,14,19)}$ In addition, the former is useful for detecting AVMs or tumors of the spinal cord. Therefore, these imaging studies should be performed to establish an early diagnosis. However, these methods cannot al-

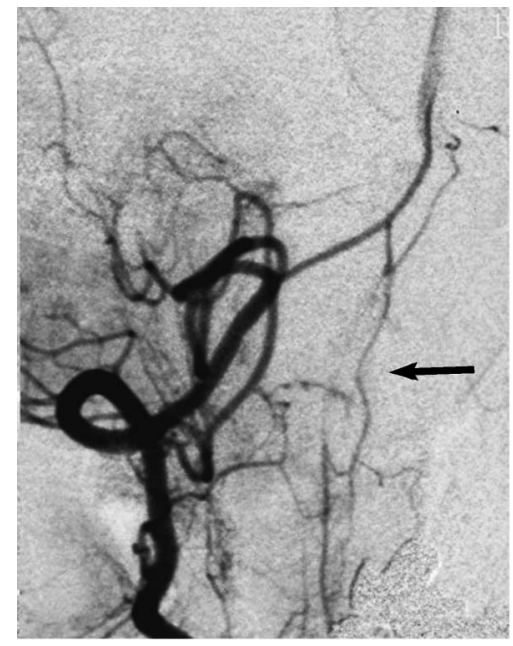

Fig. 5 Right vertebral angiogram on the 108th day showing disappearance of the lesion (arrow). ways detect abnormal findings, as shown in the present case, and are insufficient to completely confirm the lesion because of the limited sensitivity for small-caliber vessels and low flow states. ${ }^{2)}$

Symptoms caused by ruptured spinal artery aneurysms seem to correlate well with the location of the lesion (Table 2). Sudden neck pain, cessation of breathing usually accompanied by severe consciousness disturbance, and cranial nerve palsies are characteristic of aneurysms at the upper cervical levels, whereas sudden back pain and motor weakness or sensory disturbance of the lower extremities are suggestive of aneurysms at the thoracolumbar levels. Symptoms of SAH caused by spinal artery aneurysms at the cervical levels mimic those caused by cerebral artery aneurysms. In the present case, as cerebral artery aneurysms had been excluded, the severe consciousness disturbance with cardiopulmonary arrest after sudden neck pain and numbness of extremities, in conjunction with the distribution of the SAH mainly in the posterior fossa, strongly suggested that the bleeding site was at the cervical level.

The pathogenesis of spinal artery aneurysms has been previously discussed. ${ }^{15,16)}$ There are several hypotheses concerning the causal factors, such as inflammatory reaction, infection, or connective tissue disorders. ${ }^{1,3,8)}$ In contrast, spinal artery aneurysms without definite underlying disease have also been reported, as in the present case. Most of spinal artery aneurysms are small, and although both saccular and fusiform aneurysms have been identi-

Table 1 Reported cases of ruptured isolated spinal artery aneurysms

\begin{tabular}{|c|c|c|c|c|c|c|}
\hline Author (Year) & Age (yrs) & Sex & Site & Vascular supply & Operation & Outcome \\
\hline Henson and Croft $(1956)^{6)}$ & 51 & M & upper cervical part & PSA & - & death \\
\hline Garcia et al. (1979) ${ }^{3)}$ & 34 & $\mathrm{~F}$ & T6 & Adamkiewicz artery & - & death \\
\hline Yonas et al. $(1980)^{20)}$ & 42 & $\mathrm{~F}$ & upper cervical part & ASA & - & death \\
\hline Moore et al. (1982) ${ }^{12)}$ & 30 & $\mathrm{~F}$ & C1 & ASA & + & alive \\
\hline Smith et al. (1986) ${ }^{17)}$ & 29 & $\mathrm{M}$ & T12-L1 & ASA & + & alive \\
\hline Saunders et al. $(1987)^{16)}$ & 48 & $\mathrm{~F}$ & $\mathrm{~T} 1$ & anterior perforators & + & alive \\
\hline Goto et al. (1988) ${ }^{5)}$ & 53 & M & $\mathrm{C} 2$ & PSA & + & alive \\
\hline Rengachary et al. (1993) ${ }^{15)}$ & 50 & $\mathrm{~F}$ & $\mathrm{~T} 12$ & radiculomedullary artery & + & alive \\
\hline Vishteh et al. $(1997)^{18)}$ & 30 & $\mathrm{M}$ & $\mathrm{T} 11$ & Adamkiewicz artery & + & alive \\
\hline Kawamura et al. (1999) ${ }^{7)}$ & 42 & M & posterior fossa & ASA & + & alive \\
\hline \multirow[t]{2}{*}{ Chen et al. $(2001)^{2)}$} & 69 & $\mathrm{~F}$ & upper cervical part & LSA & IVR, + & alive \\
\hline & 72 & $\mathrm{~F}$ & upper cervical part & LSA & IVR & alive \\
\hline Yahiro et al. (2004) $)^{19)}$ & 71 & $\mathrm{~F}$ & $\mathrm{~T} 4-\mathrm{T} 5$ & radiculomedullary artery & + & alive \\
\hline \multirow[t]{3}{*}{ Berlis et al. (2005) $)^{1)}$} & 62 & $\mathrm{~F}$ & T5 & segmental artery & - & alive \\
\hline & 48 & M & $\mathrm{T} 12$ & Adamkiewicz artery & - & alive \\
\hline & 69 & $\mathrm{~F}$ & $\mathrm{~T} 12$ & Adamkiewicz artery & - & alive \\
\hline \multirow[t]{4}{*}{ Massand et al. (2005) ${ }^{11)}$} & 30 & M & $\mathrm{T} 11$ & Adamkiewicz artery & + & alive \\
\hline & 69 & M & L1 & radicular artery & + & alive \\
\hline & 54 & $\mathrm{M}$ & $\mathrm{T} 12$ & NA & + & alive \\
\hline & 73 & $\mathrm{M}$ & T6-T7 & radicular artery & + & alive \\
\hline Koçak et al. (2006) ${ }^{9)}$ & 54 & $\mathrm{~F}$ & $\mathrm{C} 2$ & PSA & - & death \\
\hline Nemecek et al. $(2006)^{13)}$ & 55 & $\mathrm{M}$ & $\mathrm{T} 12$ & PSA & + & alive \\
\hline Longatti et al. (2008) ${ }^{10)}$ & 54 & $\mathrm{~F}$ & $\mathrm{~T} 12, \mathrm{~T} 9$ & ASA & - & alive \\
\hline Klingler et al. (2009) ${ }^{8)}$ & 46 & $\mathrm{~F}$ & C3 & radiculomedullary artery & - & alive \\
\hline Pollock et al. (2009) ${ }^{14)}$ & 55 & $\mathrm{~F}$ & $\mathrm{C} 1-\mathrm{C} 2$ & ASA & + & alive \\
\hline Present case & 51 & $\mathrm{M}$ & C1 & ASA & - & alive \\
\hline
\end{tabular}

ASA: anterior spinal artery, IVR: interventional radiology, LSA: lateral spinal artery, NA: not available, PSA: posterior spinal artery. 
Table 2 Specific symptoms of ruptured spinal artery aneurysms by location

\begin{tabular}{|c|c|}
\hline $\begin{array}{l}\text { Location of } \\
\text { aneurysm } \\
\text { (no. of cases) }\end{array}$ & Symptoms (no. of cases) \\
\hline C1 to C3 (11) & $\begin{array}{l}\text { neck pain (3) } \\
\text { cessation of breathing (3) } \\
\text { loss of consciousness (2) } \\
\text { VIth cranial nerve palsies (2) } \\
\text { right hemiparesis (2) } \\
\text { quadriplegia (1) } \\
\text { photophobia (1) } \\
\text { lt lower extremity weakness (1) }\end{array}$ \\
\hline T4 to T7 (4) & $\begin{array}{l}\text { paraparesis or paraplegia ( } 3 \text { ) } \\
\text { sensory disturbance related to segmental levels (2) } \\
\text { back pain (2) } \\
\text { precordial pain (1) } \\
\text { numbness and pain of upper extremities (1) } \\
\text { bladder and bowel disturbance (1) } \\
\text { lt lower extremity weakness (1) }\end{array}$ \\
\hline T11 to L1 (10) & $\begin{array}{l}\text { back pain (8) } \\
\text { paraparesis or paraplegia (4) } \\
\text { paresthesia of lower extremities (3) } \\
\text { abdominal pain (3) } \\
\text { sensory disturbance related to segmental levels (2) } \\
\text { neck pain (1) } \\
\text { tetraplegia (1) } \\
\text { bladder and bowel disturbance (1) } \\
\text { lt lower extremity weakness (1) }\end{array}$ \\
\hline
\end{tabular}

fied, the latter are more frequent. ${ }^{1-3,5,8-10,12-15,18,19)}$ Three cases of spinal artery aneurysms were found to be fusiform, dissecting lesions at surgery. ${ }^{11)}$ Another ruptured posterior spinal artery aneurysm was confirmed to be a dissecting lesion by histopathological analysis. ${ }^{13)}$ Therefore, some spinal artery aneurysms appear to be dissecting lesions. In the present case, spontaneous disappearance of the lesion suggests a dissecting aneurysm. Although several factors may contribute to the formation of aneurysms, hemodynamic stress seems highly likely to be the principal etiological factor. ${ }^{1,7,10)}$ This assumption is based on previous reports postulating that spinal artery aneurysms often occur with AVMs or coarctation of the aorta. High blood flow caused by AVMs and collateral routes induced by coarctation of the aorta have been considered to be significant etiological factors for the formation of aneurysms. ${ }^{10,15)}$ Hemodynamic stress caused by bilateral vertebral artery occlusions was the etiological factor for one case of anterior spinal artery aneurysm.7) Two cases of lateral spinal artery aneurysms were probably caused by hemodynamic stress from high-grade stenosis of the vertebral artery. ${ }^{2)}$

In the present case, the anterior spinal artery and the distal portion of the right vertebral artery were not opacified on the initial DSA. However, the third DSA on the 18th day showed an anterior spinal artery aneurysm as the flow of the vertebral artery and the anterior spinal artery improved. The fourth DSA performed on the 108th day demonstrated spontaneous regression of the lesion. The reason why the right vertebral artery was insufficiently projected on the initial DSA is unclear, but may have involved early spasm, which also caused the poor projection of the anterior spinal artery. If the ascending cervical artery had been investigated as a collateral route, the anterior spinal artery might have been recognized. The reason for the spontaneous regression of the aneurysm is also unclear. Spontaneous healing of the dissection and obliteration of the aneurysm may be the most likely mechanism. ${ }^{1)}$

The therapeutic strategy for ruptured spinal artery aneurysms remains controversial. Surgical interventions, endovascular approaches, and wait-and-see strategies have been adopted. ${ }^{1,2,4,10,18)}$ Four patients died without surgery. Re-bleeding was confirmed or suspected in three of these patients. Two of these three patients died 8 hours and within 24 hours after admission, respectively. ${ }^{3,9}$ The third patient was treated conservatively and died 17 days after admission. ${ }^{20)}$ The other patient presented with SAH one week before admission and died on the 8th day after admission. ${ }^{6}$ In this case, ruptured posterior spinal artery aneurysm was confirmed at autopsy, but there was no clear episode of re-bleeding. In contrast, no deaths were reported among patients who underwent surgical or endovascular treatment. Therefore, the ideal treatment for ruptured spinal artery aneurysms appears to be surgical or endovascular intervention at the acute stage to prevent fatal re-bleeding. However, several factors should be taken into consideration when planning a surgical approach. Most spinal artery aneurysms are small and fusiform along the course of the parent artery, lacking a surgical neck. ${ }^{1,4,15)}$ If distal flow to the lesion is present, the parent artery must be preserved, and direct microsurgical reconstruction may be required. In particular, surgical access is difficult for anterior spinal artery aneurysms located at the upper cervical levels because of the narrow operative view. Direct surgery for such lesions was performed in three cases. ${ }^{7,12,14)}$ The surgical procedures included a suboccipital approach with or without cervical laminectomy and a far lateral approach to the cervicomedullary junction. Obliteration by clipping was performed in two cases, and resection with trapping was performed in the other case. The postoperative courses were favorable in all cases. The endovascular approach may be an appropriate choice, especially if surgical intervention is difficult considering the location of the lesion or the condition of the patient. However, the lesion cannot always be reached endovascularly, and the reported number of cases is too small to validate its efficacy. ${ }^{2)}$ In contrast, Longatti et al. ${ }^{10)}$ preferred conservative therapy because of the small size of the artery, the presence of distal flow, and the absence of cord compression. They considered the mass effect of aneurysms or surrounding blood clots to be the only indication for surgical treatment. Likewise, a wait-and-see strategy was recommended because of the possibility of spontaneous regression. ${ }^{11}$ These reported cases, as well as the present case, had a good outcome after the wait-andsee strategy. Thus, conservative management is another therapeutic choice for ruptured spinal artery aneurysms, especially if the neurological and general conditions of the patients are too critical to tolerate early surgical or endovascular intervention. If a wait-and-see strategy is chos- 
en, strict control of blood pressure must be maintained to prevent re-bleeding, and DSA must be repeated to observe the morphological change of the lesion, because the natural history of ruptured spinal artery aneurysms has yet to be elucidated.

Isolated spinal artery aneurysms manifesting as $\mathrm{SAH}$ are extremely rare. However, if SAH of unknown etiology is encountered, spinal artery aneurysm should be considered as a potential bleeding source. Despite the controversy concerning the treatment strategy, ruptured spinal artery aneurysms can be treated conservatively because of the possibility of spontaneous regression demonstrated in previous cases, as well as in the present case. Follow-up angiography is required to evaluate the natural course of the lesion, and to establish the therapeutic strategy.

\section{References}

1) Berlis A, Scheufler KM, Schmahl C, Rauer S, Götz F, Schumacher M: Solitary spinal artery aneurysms as a rare source of spinal subarachnoid hemorrhage: potential etiology and treatment strategy. AJNR Am J Neuroradiol 26: 405-410, 2005

2) Chen CC, Bellon RJ, Ogilvy CS, Putman CM: Aneurysms of the lateral spinal artery: report of two cases. Neurosurgery 48: 949-954, 2001

3) Garcia CA, Dulcey S, Dulcey J: Ruptured aneurysm of the spinal artery of Adamkiewicz during pregnancy. Neurology 29: 394-398, 1979

4) Gonzalez LF, Zabramski JM, Tabrizi P, Wallace RC, Massand MG, Spetzler RF: Spontaneous spinal subarachnoid hemorrhage secondary to spinal aneurysms: diagnosis and treatment paradigm. Neurosurgery 57: 1127-1131, 2005

5) Goto Y, Kamijyo Y, Yonekawa Y, Kikuchi H: Ruptured aneurysm of the posterior spinal artery of the upper cervical spinal cord: case report. Neurosurgery 22: 558-560, 1988

6) Henson RA, Croft PB: Spontaneous spinal subarachnoid hemorrhage. Q J Med 97: 53-66, 1956

7) Kawamura S, Yoshida T, Nonoyama Y, Yamada M, Suzuki A, Yasui N: Ruptured anterior spinal artery aneurysm: a case report. Surg Neurol 51: 608-612, 1999
8) Klingler JH, Gläsker S, Shah MJ, Van Velthoven V: Rupture of a spinal artery aneurysm attributable to exacerbated Sjögren syndrome: case report. Neurosurgery 64: E1010-1011, 2009

9) Koçak A, Ateş Ö, Çayli SR, Saraç K: Isolated posterior spinal artery aneurysm. Br J Neurosurg 20: 241-244, 2006

10) Longatti P, Sgubin D, Di Paola F: Bleeding spinal artery aneurysms. Case report. J Neurosurg Spine 8: 574-578, 2008

11) Massand MG, Wallace RC, Gonzalez LF, Zabramski JM, Spetzler RF: Subarachnoid hemorrhage due to isolated spinal artery aneurysm in four patients. AJNR Am J Neuroradiol 26: 2415-2419, 2005

12) Moore DW, Hunt WE, Zimmerman JE: Ruptured anterior spinal artery aneurysm: repair via a posterior approach. Neurosurgery 10: 626-630, 1982

13) Nemecek AN, Sviri G, Hevner R, Ghodke B, Britz GW: Dissecting aneurysm of the thoracic posterior spinal artery. Case illustration. J Neurosurg Spine 5: 555, 2006

14) Pollock JM, Powers AK, Stevens EA, Sanghvi AN, Wilson JA, Morris PP: Ruptured anterior spinal artery aneurysm: a case report. J Neuroimaging 19: 277-279, 2009

15) Rengachary SS, Duke DA, Tsai FY, Kragel PJ: Spinal arterial aneurysm: case report. Neurosurgery 33: 125-130, 1993

16) Saunders FW, Birchard D, Willmer J: Spinal artery aneurysm. Surg Neurol 27: 269-272, 1987

17) Smith BS, Penka CF, Erickson LS, Matsuo F: Case report: subarachnoid hemorrhage due to anterior spinal artery aneurysm. Neurosurgery 18: 217-219, 1986

18) Vishteh AG, Brown AP, Spetzler RF: Aneurysm of the intradural artery of Adamkiewicz treated with muslin wrapping: technical case report. Neurosurgery 40: 207-209, 1997

19) Yahiro $T$, Hirakawa $K$, Iwaasa $M$, Tsugu $H$, Fukushima $T$, Utsunomiya $\mathrm{H}$ : Pseudoaneurysm of the thoracic radiculomedullary artery with subarachnoid hemorrhage. Case report. J Neurosurg 100(3 Suppl Spine): 312-315, 2004

20) Yonas H, Patre S, White RJ: Anterior spinal artery aneurysm. Case report. J Neurosurg 53: 570-573, 1980

Address reprint requests to: Jun Karakama, M.D., Department of Neurosurgery, Tokyo Medical and Dental University, 1-5-45 Yushima, Bunkyo-ku, Tokyo 113-8519, Japan. e-mail: sofas_float@yahoo.co.jp 